\title{
NeuroImage
}

ELSEVIER

www.elsevier.com/locate/ynimg

NeuroImage 24 (2005) 802-809

\section{Lateral modulation of BOLD activation in unstimulated regions of the human visual cortex}

\author{
Chien-Chung Chen, ${ }^{\mathrm{a}, \mathrm{b}, *}$ Christopher W. Tyler, ${ }^{\mathrm{a}}$ Chia-Li Liu, ${ }^{\mathrm{b}, \mathrm{c}}$ and Yao-Hong Wang ${ }^{\mathrm{c}}$ \\ ${ }^{a}$ The Smith-Kettlewell Eye Research Institute, San Francisco, CA 94115, USA \\ ${ }^{\mathrm{b}}$ Department of Psychology, National Taiwan University, Taipei 106, Taiwan \\ ${ }^{\mathrm{c}}$ The MRI/MRS Laboratory, National Taiwan University, Taipei 106, Taiwan
}

Received 25 May 2004; revised 8 September 2004; accepted 15 September 2004

Available online 21 November 2004

\begin{abstract}
After staring at a blank region surrounded by a dynamic background for a few seconds, observers report a twinkle aftereffect in the unstimulated blank region. The significance of this twinkle aftereffect is that it occurs at a location that received no stimulation, and therefore reflects a rebound from lateral inhibition within the dynamic processing system. To study this inhibitory rebound effect, the blood oxygenation level dependent (BOLD) activation in the visual cortex was measured while the observers were viewing a flickering pin-wheel pattern alternating with a blank test. Retinotopic regions corresponding to the inter-wedge regions in the pin-wheel pattern showed activation negatively correlated with the test sequence. While the BOLD activation in the visual cortex is generally considered to be retinotopically driven by the visual stimuli, we were able to show a sustained negative activation in the unstimulated regions, with properties that correspond to those of the inhibitory rebound of the perceived aftereffect.
\end{abstract}

(C) 2004 Elsevier Inc. All rights reserved.

Keywords: BOLD activation; Visual cortex; Human

\section{Introduction}

Functional magnetic resonance imaging (fMRI) studies measure the blood oxygenation level dependent (BOLD) activation that derives from neuronal activity driven by changes in sensory stimulation or cognitive tasks. In fMRI of the visual cortex, the stimulus-driven BOLD activation has two aspects. First, in space, the BOLD activation in the early visual cortex shows a retinotopic property (Engel et al., 1997; Tootell et al., 1998). That is, the activation of a particular voxel corresponds to the presence of visual stimuli at a certain location. This retinotopic mapping

* Corresponding author. Department of Psychology, National Taiwan University, 1, Sec 4, Roosevelt Road, Taipei 106, Taiwan.

E-mail address: chen@ski.org (C.-C. Chen).

Available online on ScienceDirect (www.sciencedirect.com). property, which maps the retinal image locations to their corresponding cortical activation areas, has been a standard procedure in visual fMRI. However, to make the retinotopic mapping possible, a cortical area not only has to show an increment in activation when a stimulus falls on its corresponding retinal location but also keep activation at the baseline level when stimuli are presented to other parts of retina. That is, an implication of the retinotopic mapping experiment is that, except for some random fluctuation, the unstimulated cortical areas exhibit no activation change from the baseline.

The second aspect of the stimulus-driven property is that, in time, the BOLD activation is positively correlated with the stimulus onset. When presented with a visual stimulus, the BOLD activation in the responsive cortical area shows an increase shortly after the stimulus onset and a post-stimulation undershoot after the stimulus offset (e.g., Kwong et al., 1992). The theoretical hemodynamic impulse response functions also show a similar biphasic form (Aubert and Costalat, 2002; Buxton et al., 1998). Hence, in a block design experiment, where a test condition alternates with a control condition, the BOLD activation should be expected to increase in the test condition and decrease in the control condition. Therefore, allowing for a short hemodynamic delay, the cortical areas responsive to the test stimulus should have a BOLD activation that is positively correlated with the presence of the test.

Recent reports of sustained negative BOLD responses (e.g., Shmuel et al., 2002) may challenge the stimulus-driven assumption. It is possible to have a decrement of BOLD activation that follows the onset of the stimulus and has a duration similar to that of the positive BOLD activation. The cause of the sustained negative BOLD activation is not clear, however. It could be due to an increment in neural activity, hence an increment in oxygen consumption $\left(\mathrm{CMRO}_{2}\right)$ without compensation in cerebral blood flow (CBF). In this scenario, the sustained negative BOLD activation is just a prolonged version of the initial dip, a small and brief negative activation that occurs immediately after the stimulus onset observed in high field magnets (Menon et al., 1995). 
The sustained negative BOLD activation could also be a decrement in CBF followed by a change of neural activity. In this scenario, the mechanism underlying the sustained negative BOLD activation is similar to the positive BOLD activation but working in the opposite direction. For instance, it has been shown that the BOLD activation is positively correlated with local field potentials (Logothetis et al., 2001). In the positive activation, an increment in field potentials prompts an increment of CBF. Conversely, in the negative activation, the decrement of field potential caused by, say, neural lateral inhibition, may lead to a decrement in CBF. However, the sustained negative BOLD activation could also be of hemodynamic origin that is unrelated to the neural activity within the voxels showing negative activation. For instance, an increment of $\mathrm{CBF}$ in some cortical region may result in a redistribution of blood and a decrement of $\mathrm{CBF}$ in neighboring cortical regions. This blood steal could produce a decrement in BOLD activation in the corresponding voxels.

Röther et al. (2002) showed that on a patient with extracranial artery disease, a sustained negative BOLD activation could be created by an increment in $\mathrm{CMRO}_{2}$ without $\mathrm{CBF}$ compensation and thus showed the same mechanisms that underlie the initial dip can be sustained for longer durations. In normal observers, however, the sustained negative BOLD activation is different from the initial dip as follows. In space, the cortical areas showing initial dip are found to lie within the areas that show positive BOLD activation ( $\mathrm{Hu}$ et al., 1997) while the areas showing the sustained negative BOLD activation are reported outside the areas for positive BOLD activation. In time, the initial dip is brief, usually lasts less than 4 s (Hu et al., 1997) while the sustained BOLD activation may last as long as the positive BOLD activation. In addition, the size of the initial dip is small and is often difficult to detect, while the sustained negative lobe is ubiquitous.

The contrast between the sustained negative BOLD activation and the initial dip is even more pronounced in a block design experiment. Given the common parameters used in a block experiment, repetition time (TR) of 2-4 s and epoch duration of $15-30 \mathrm{~s}$, the initial dip will produce no noticeable effect on the time course of the activation. On the other hand, the sustained negative BOLD activation will produce an activation time series that is negatively correlated with the test epochs. Recently, Shmuel et al. (2002) were able to rule out the hypothesis that the sustained negative BOLD activation is related to the initial dip. They suggested that the reduction of neural activity can be a cause of the sustained negative BOLD activation but they could not rule out a contribution from the hemodynamics of blood-steal effects. It is possible that the negative BOLD may contain both neural and hemodynamic components. The sustained negative BOLD activation reported by Shmuel et al. (2002) does not seem to relate to the visual percept. There is no published report of a visual artifact around the visual stimulus. Hence, the lack of visual percept correspondence may be used as an argument against the neural origin of sustained negative BOLD.

We therefore addressed the issue of whether the neural activity and the blood-steal hypothesis may be differentiated in the visual cortex. We used an experimental paradigm that, in addition to the positive activation, produces visual percepts positively correlated with the negative BOLD activation. The rationale is that if the sustained negative BOLD activation is due to the blood steal and is independent of the neural activity, it should be of the same magnitude regardless of the occurrence of an induced aftereffect. On the other hand, if the sustained negative BOLD activation is due to the reduction of neural activity, it should be reduced under conditions where reduced inhibition results in a reduced visual percept.

Here, we demonstrate a sustained negative BOLD activation in the unstimulated brain regions that is correlated with the human visual percept. The experiment is based on known lateral interactions involved in the twinkle aftereffect. After staring at a grey region surrounded by a dynamic patterned background (adapter), observers perceive a twinkling aftereffect in the location of the grey region when the pattern stimulus is removed (Hardage and Tyler, 1995; Ramachandran and Gregory, 1991). That is, the aftereffect is induced in a region that had never received any stimulation during either the adapting or the test phases and must therefore result from the lateral interaction between the mechanisms responding to the dynamic surround region and those in the blank test region. In particular, Hardage and Tyler were able to dissociate the aftereffect from the filling-in explanation advocated by Ramachandran and Gregory, since the twinkle aftereffect is found under many conditions in which no filling-in occurs. Here we show that the negative BOLD activation is positively correlated with the aftereffect. In addition, we show that the temporal characteristics of the negative BOLD activation are different from those of the positive activation in a form that is incompatible with the hemodynamic hypothesis. Hence, at least some aspects of the negative activation must have a neural origin.

\section{Methods \\ Stimulus and the experimental design}

The adapting stimulus was a pinwheel pattern containing four $45^{\circ}$ checkerboard wedges separated from each other by $45^{\circ}$ blank regions set at the mean luminance and extended $4.5^{\circ}$ from the central fovea (Fig. 1). In the main experiment, the checkerboards were counterphase flickering at $10 \mathrm{~Hz}$ during the presentation. The 21-s adapter sequence alternated with a 21 -s blank field, which contained only a fixation mark, forming a 42-s period, with six periods in each scan session. In addition, a 21-s blank period was inserted in the middle and at the end of the session to establish the baseline unstimulated level. The control experiment was the same as the main experiment except that the flicker rate of the checkerboards was reduced to $1 \mathrm{~Hz}$.

The cortical regions retinotopically corresponding to the stimulated wedges and (conversely) to the inter-wedge regions were determined by a spatial localizer. The spatial localizer contained two patterns: one being the same as the adapter and the other being the same as the adapter but rotated clockwise by $45^{\circ}$. The two patterns in the spatial localizer alternated with each other for $21 \mathrm{~s}$ to form a 42-s period for six periods. Both patterns contained checkerboards counter-phase flickering at $10 \mathrm{~Hz}$. Six observers with normal vision (four females, two males) participated in the study, consisting of five volunteers and one of the authors.

\section{Data acquisition and analysis}

The images were collected with a Bruker 3-T scanner located at National Taiwan University. A high-resolution anatomical (T1weighted) MRI volume scan of the entire head was run once on each observer (voxel size $=1 \times 1 \times 2 \mathrm{~mm}$ ). Within each scanning session, both functional (T2*-weighted, BOLD) responses and anatomical (T1-weighted) images were acquired in identical planes. The images were collected in 18 transverse planes parallel 

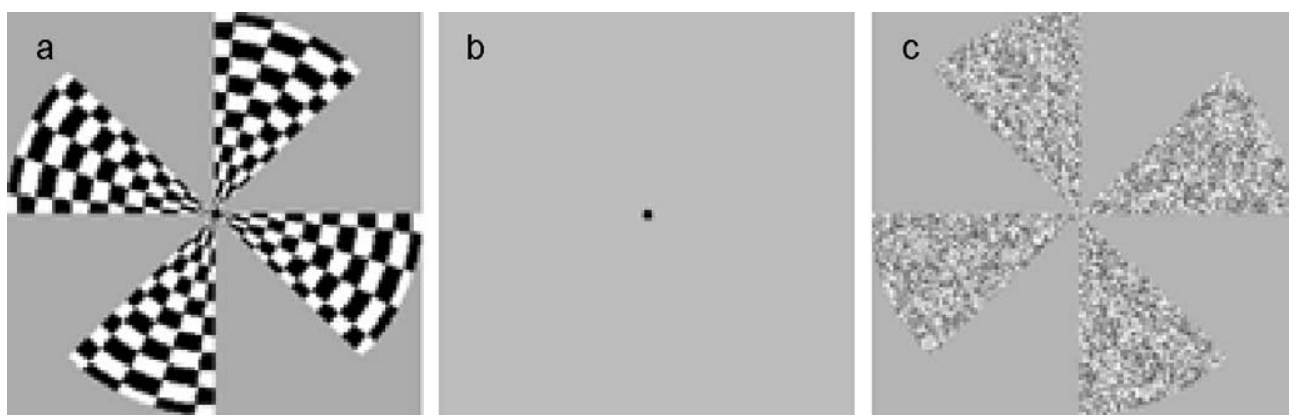

Fig. 1. Stimuli used in the experiment. The adapter (a) was a pin-wheel pattern consisting of four checkerboard wedges counter-phase flickering at $10 \mathrm{~Hz}$ (1 $\mathrm{Hz}$ in the control experiment). The adapter was alternated with a blank test (b) that contained only a fixation point. All observers reported perceiving a twinkling afterimage [illustrated in (c)] in the inter-wedge regions of the adapter while viewing the blank test.

to the AC-PC (anterior commissure-posterior commissure) line. An echo-planar imaging sequence (Stehling et al., 1991) was used to acquire the functional data $(\mathrm{TR}=3500 \mathrm{~ms}, \mathrm{TE}=35 \mathrm{~ms}$, flip angle $=90^{\circ}$ voxel resolution $\left.=2.34 \times 2.34 \times 3 \mathrm{~mm}\right)$. The main experiment lasted $304.5 \mathrm{~s}$ (87 images). The first $10.5 \mathrm{~s}$ (three images) was excluded from further analyses. Thus, the data analyzed for each scan spanned 294 s (84 images). We used SPM (Friston et al., 1995) to realign the EPI images acquired in the spatial location, the main and the control experiment. The EPI images were coregistered with the T1-weighted image with a
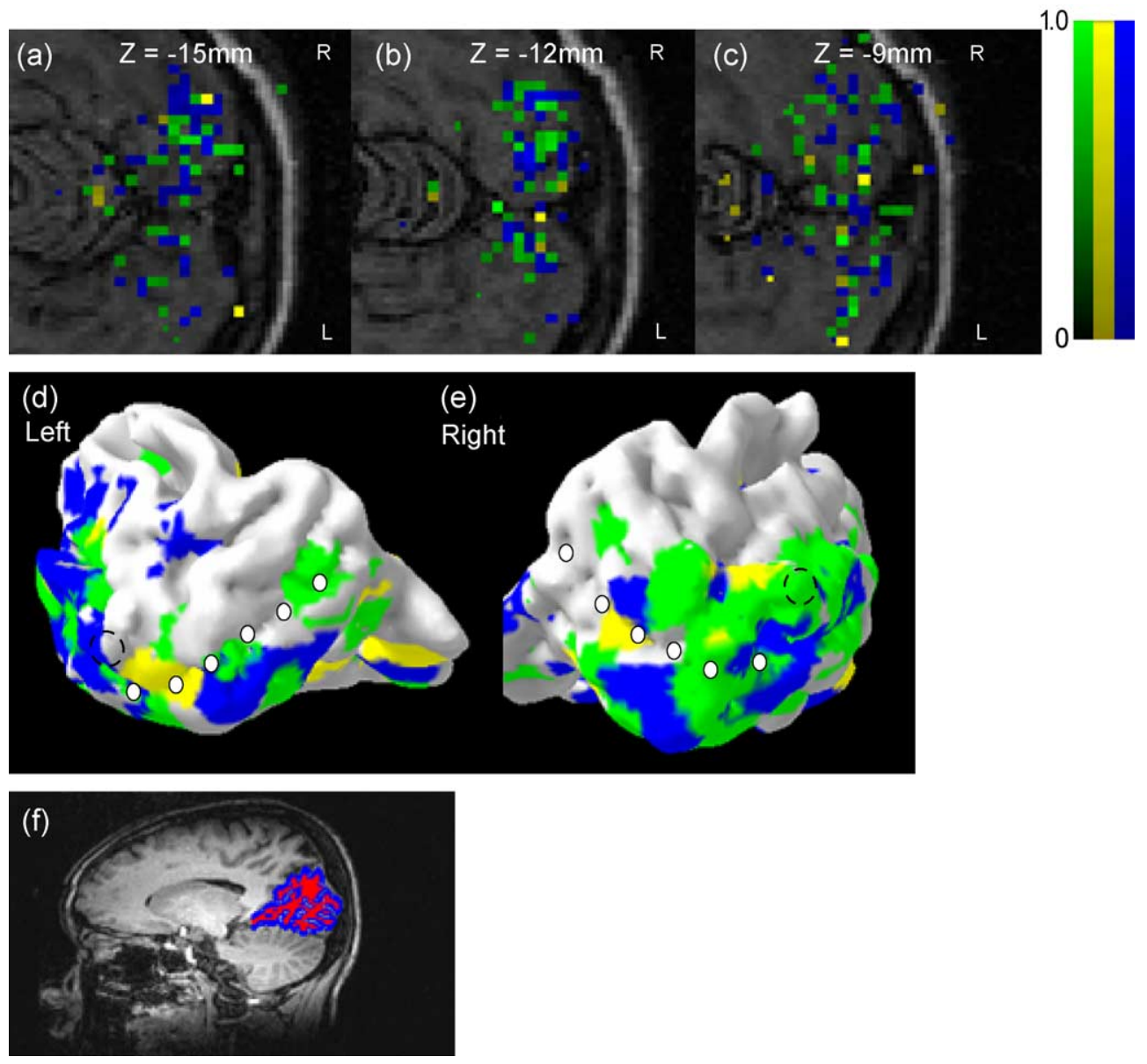

Fig. 2. A typical activation map for the twinkle aftereffect with 10-Hz adapter. (a)-(c) show activation on three axial planes with depth $z=-15,-12$ and -9 $\mathrm{mm}$ in Talairach coordinates (Talairach and Tournoux, 1988). (d) and (e) show activation on a 3D rendered surface of the left (L) and right (R) occipital cortex to give a clear view of the spatial relation among activated voxels. (f) shows the gray matter (blue) and the white matter (red) of the rendered area in (d) and (e) for reference. In (a)-(e), colored patches denote voxels showing significant activation change $(|r|>0.35)$ in the experiment (for this observer, $18.1 \%$ of all voxels identified by the spatial localizer). Green: activated voxels in the stimulated wedge regions; blue: voxels in the inter-wedge regions with activation negatively correlated with the experimental sequence; yellow: voxels in the inter-wedge regions with positively correlated with the experimental sequence. For this observer, $66 \%$ of the significantly activated inter-wedge voxels showed a negative correlation with the experimental sequence (blue). The white dots mark the calcarine sulcus and the black open circles mark the occipital pole in (d) and (e). 
publicly available software (Wandell et al., 2000), which also was used to perform the data analysis and the $3 \mathrm{D}$ rendering of Figs. 2 and 5. Statistical analysis of the BOLD activation was based on linear correlation between the time series and the experimental sequence (Engel et al., 1997).

\section{Results and discussion}

\section{BOLD activation to twinkle aftereffect}

Our experiment used a block design in which the high-contrast counter-phase checkerboard adapter was a pinwheel pattern lasting $21 \mathrm{~s}$ that alternated with a 21-s uniform blank test (see Methods). All observers verbally reported some form of twinkle aftereffect in the inter-wedge regions when viewing the uniform blank test field following the 21-s adaptation period.

The typical behavior depicted in Fig. 2 illustrates that a substantial proportion of the inter-wedge voxels showed a significant BOLD activation change (correlation coefficient $|r|>$ 0.37 , two-tailed $P=0.001$ ) between the adapting and the test epochs, even though those voxels corresponded to retinal areas that were never stimulated during the experiment. The cortical regions corresponding retinotopically to the wedge and interwedge regions in the adapter was identified by a spatial localizer (see Methods). In the majority $\left[55-72 \%, \chi^{2}(1)=12.02-\right.$ $136.80, P<5 \times 10^{-4}$ across observers or on average, $60 \%, \chi^{2}(1)=$
95.97, $P<1.4 \times 10^{-16}$ ] of voxels, the unstimulated activation was in counter-phase with the adapter onset (blue patches in Fig. 2): The BOLD signal decreased when the adapter appeared and increased following its disappearance. A minority of the voxels showed unstimulated activation that was in phase with the stimulus onset (yellow patches in Fig. 2), presumably corresponding to spread of the activation from the stimulated regions. The wedgeregion voxels that showed significant in-phase activation changes in the experiment are denoted as green spots in Fig. 2 as reference points. It can be seen that all the yellow patches lie between the green stimulated and the blue counter-phase regions, consistent with the spread of activation hypothesis.

An analysis of the time series reveals both the suppression and rebound of BOLD activation in the counter-phase inter-wedge voxels. The BOLD activation decreased with the onset of the adapter and increased with the offset (Fig. 3a), in contrast to the wedge region voxels (Fig. 3c) where activation increased with the onset of the adapter. The two extended blank periods in the middle and the end of the scan were placed to establish the base line. The activation of wedge region voxels returned to a resting level during this period while the inter-wedge voxels showed recovery only toward the end of this period. This qualitative result implies that there were different time courses for the counter-phase and inphase responses.

To further analyze the response time course, we fit the activation time series by convolving the complete experimental stimulation sequence with a parameterized BOLD impulse
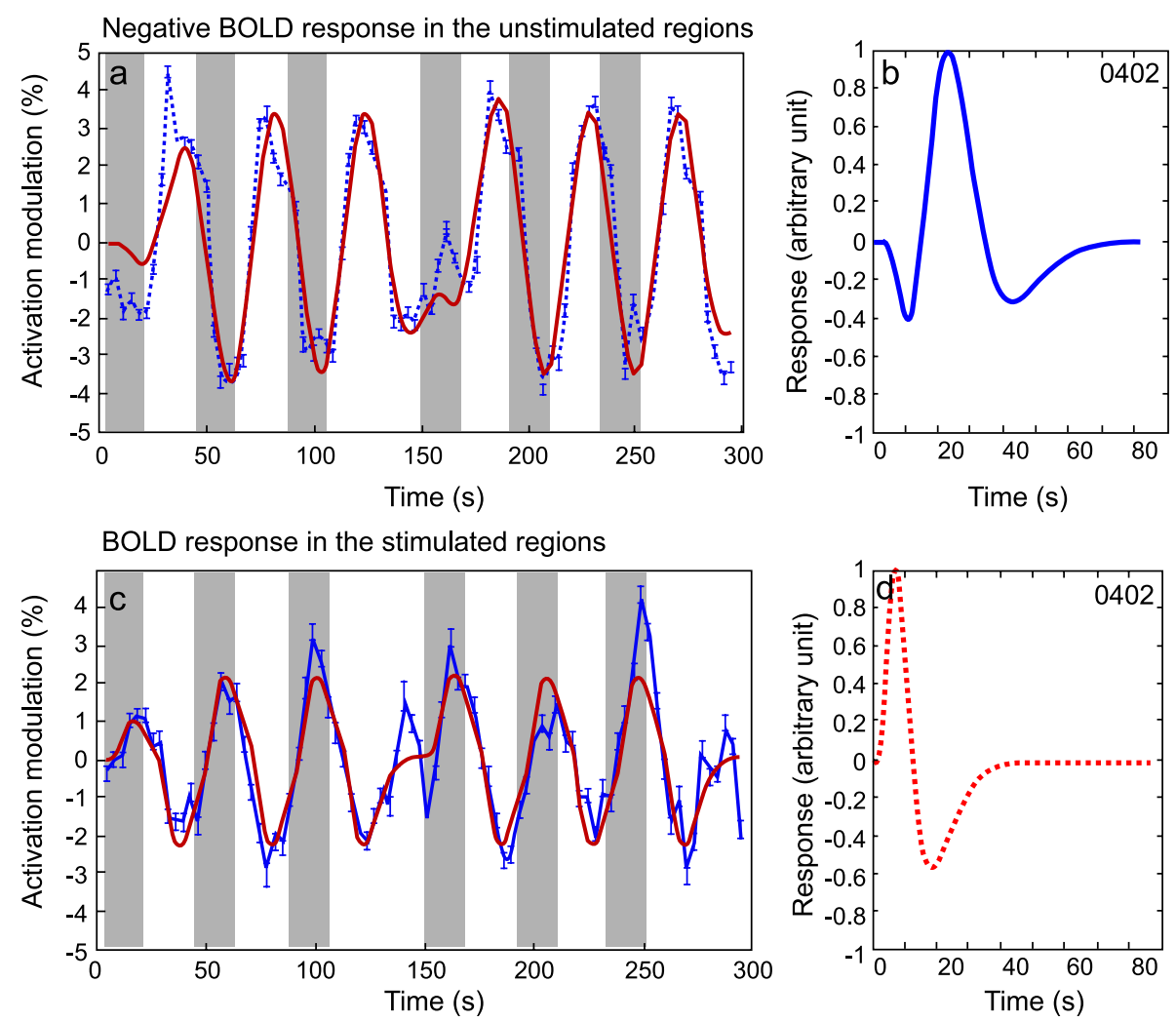

Fig. 3. (a) Averaged percentage change of BOLD activation time series for inter-wedge voxels showing negative correlation with the stimulation periods (grey bars) relative to the blank screen periods (white bars) and (b) is the best fit impulse response function to this time series. (c) Averaged percentage change of BOLD activation time series for wedge voxels showing positive correlation with the stimulation periods and (d) is the best fit impulse response function to this time series. The error bars denote 1 standard error of the means. The smooth curve is the fit of a convolution of the experimental sequences with a difference-ofgamma impulse response function: $w_{1}\left(x / \alpha_{1}\right)^{\beta_{1}} \mathrm{e}^{-x / \alpha 1}-w_{2}\left(x / \alpha_{2}\right)^{\beta 2} \mathrm{e}^{-x / \alpha 2}$ where $w_{i}, \alpha_{i}$ and $\beta_{i} ; i=1,2$ are free parameters. 
Table 1

Parameters for the difference-of-gamma response functions

\begin{tabular}{|c|c|c|c|c|c|c|c|}
\hline & & \multicolumn{6}{|c|}{ Observers } \\
\hline & & 0129 & 0312 & 0320 & 0326 & 0402 & 0409 \\
\hline \multirow[t]{6}{*}{ Positive activation } & $w_{1}$ & 260.06 & 191.28 & 2.54 & 5.27 & 6.85 & 13.87 \\
\hline & $\alpha_{1}$ & 1.30 & 0.91 & 0.34 & 0.69 & 0.46 & 0.41 \\
\hline & $\beta_{1}$ & 3.42 & 2.46 & 5.69 & 5.42 & 5.39 & 4.72 \\
\hline & $w_{2}$ & 61.32 & 117.36 & $8 E-6$ & 0.055 & 2.08 & 1.35 \\
\hline & $\alpha_{2}$ & 0.97 & 2.80 & 0.45 & 0.75 & 0.82 & 1.03 \\
\hline & $\beta_{2}$ & 4.7 & 1.75 & 22.84 & 8.02 & 5.84 & 5.72 \\
\hline \multirow[t]{6}{*}{ Negative activation } & $w_{1}$ & $3 E-6$ & $4 \mathrm{E}-5$ & 0.02 & $4 \mathrm{E}-5$ & 0.05 & $7 E-9$ \\
\hline & $\alpha_{1}$ & 0.50 & 0.69 & 0.60 & 0.64 & 0.84 & 0.48 \\
\hline & $\beta_{1}$ & 13.02 & 12.07 & 8.67 & 11.84 & 8.84 & 14.85 \\
\hline & $w_{2}$ & 9.34 & 0.0743 & 102.10 & 5.574 & 5.4361 & 22.47 \\
\hline & $\alpha_{2}$ & 1.13 & 1.02 & 2.19 & 1.33 & 1.25 & 1.97 \\
\hline & $\beta_{2}$ & 4.69 & 8.58 & 2.34 & 5.85 & 6.21 & 3.67 \\
\hline
\end{tabular}

response function defined as the difference of two gamma functions defined by:

$\mathrm{d} g(x)=w_{1}\left(x / \alpha_{1}\right)^{\beta_{1}} \mathrm{e}^{-x / \alpha_{1}}-w_{2}\left(x / \alpha_{2}\right)^{\beta_{2}} \mathrm{e}^{-x / \alpha_{2}}$

where $w_{i}, \alpha_{i}$ and $\beta_{i}, i=1,2$ are free parameters. Among those parameters, $\alpha_{i}$ determines the position of the peak of the $i$ th component in time, $\beta_{i}$ determines the steepness of response change in the $i$ th component and $w_{i}$ is a parameter of arbitrary units that determines the maximum response change of the $i$ th component. This function incorporates sufficient parameterization to capture all the features of the impulse responses derived from the wellestablished "balloon model" of Buxton et al. (1998). The parameters of the two Gamma functions were optimized separately for each observer for best fit to the respective data sets. The bestfiting difference-of-gamma parameters for each observer are listed in Table 1. For a better comparison across observers, the fitted impulse response functions were then normalized to a maximum response of 1, as shown in Figs. 3 and 4. The in-phase voxels in the stimulated regions showed a typical biphasic BOLD response function, beginning with an increase followed by a decrease. The individual in-phase impulse response functions are plotted upside down as the dashed red curves in Fig. 4 for ready comparison with the corresponding counter-phase functions. For the counter-phase voxels, all observers required a triphasic impulse response to fit the BOLD time sequence (solid blue curves, Fig. 3b) with an early reduction followed by a slow increase and then another dip. The size of the second dip reached 0.25 on average, which is statistically significantly different from zero $[t(5)=8.831, P=$ $0.00031<0.05]$. The triphasic impulse response functions were also fitted with the same family of difference-of-gamma functions as the in-phase voxels but with different parameters.
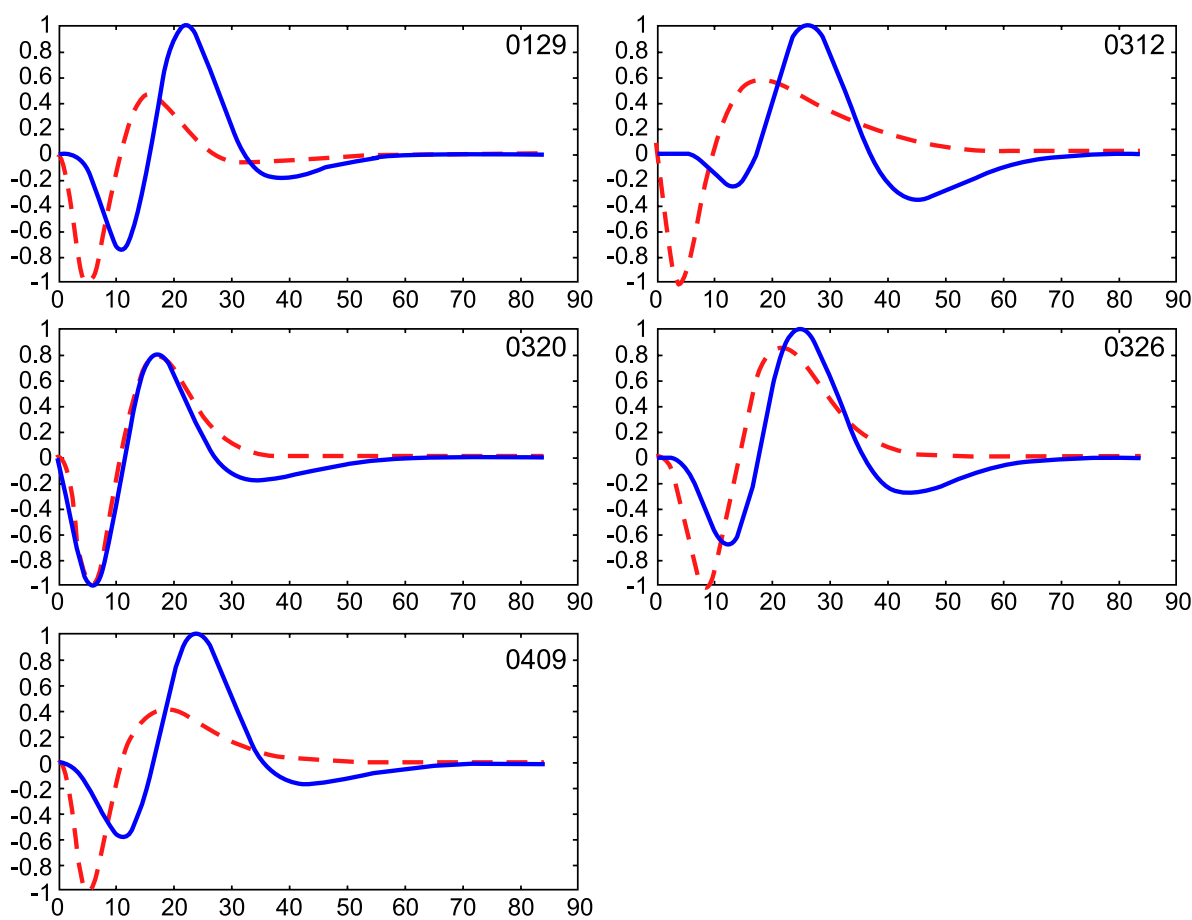

Fig. 4. The impulse response functions for counter-phase voxels (solid curves), and in-phase voxels (dashed curves) for five observers. The in-phase curves are plotted upside down to give a better comparison of the two functions. The response functions are normalized such that the absolute value of the maximum response for each function is 1 . 
Table 2

"Landmarks" on the response functions

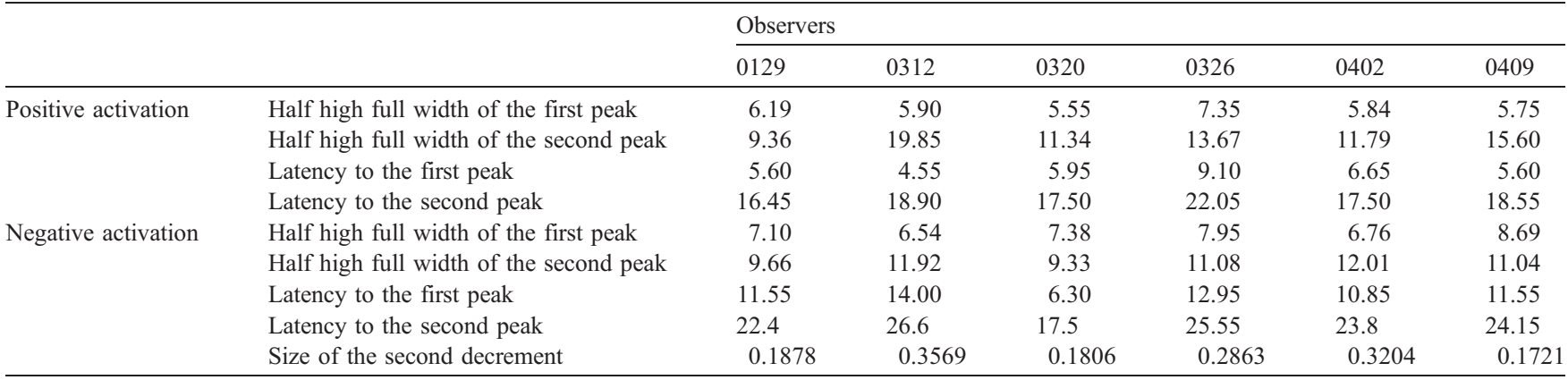

Quantitative comparisons of the time courses for the in-phase and counter-phase impulse response functions are provided in Table 2. The counter-phase response functions have a slower start than the inphase ones. On average, it took $6.2 \mathrm{~s}$ for the in-phase response to reach the first positive peak response but $11.2 \mathrm{~s}$ for the counter-phase response to reach its first peak (maximum suppression). The time to reach the second peak, on average, is $18.49 \mathrm{~s}$ for the in-phase response function but $23.3 \mathrm{~s}$ for the counter-phase response. These differences are statistically significant, with pairwise $t(5)=4.04$ $(P=0.005<0.01)$ for the first peak delay and $t(5)=4.34$ $(P=0.0037<0.01)$ for the second. However, the responses of the counter-phase voxels are actually more transient than the first. The full width at half-height for the second peak response is significantly narrower $[t(5)=2.1654, P=0.0413<0.05]$ in the counter-phase response functions $(10.83 \mathrm{~s})$ than the in-phase ones $(13.60 \mathrm{~s})$.

In order to determine whether the difference in in-phase and counter-phase BOLD time courses is of neural origin, we first need evaluate the role of blood steal from regions adjacent to the activated regions, which come in two forms. The differences in peak times and durations are incompatible with the idea that the negative BOLD could arise from immediate hydrostatic drainage to fill the increase in volume left when blood vessels in the neighborhood of activated neurons expand. Any such hydrostatic effects should be immediate and should result in a negative BOLD signal that is the exact inverse of the primary signal. A second hypothesis is that the blood drainage increases the flow rate of the blood through the vessels in the simulated area and that the outlying vessels in the unstimulated area respond to this flow by contracting with a delayed time course. This second hypothesis is relatively implausible because there is more reason to expect them to expand in order to deliver a greater volume of blood to the activated area. In addition, it would predict the inverse of the negative BOLD response is a delayed version of the positive BOLD response. This prediction is not consistent with the briefer third peak of the negative BOLD response in our data. There is thus no support for either form of the blood-drainage hypothesis.
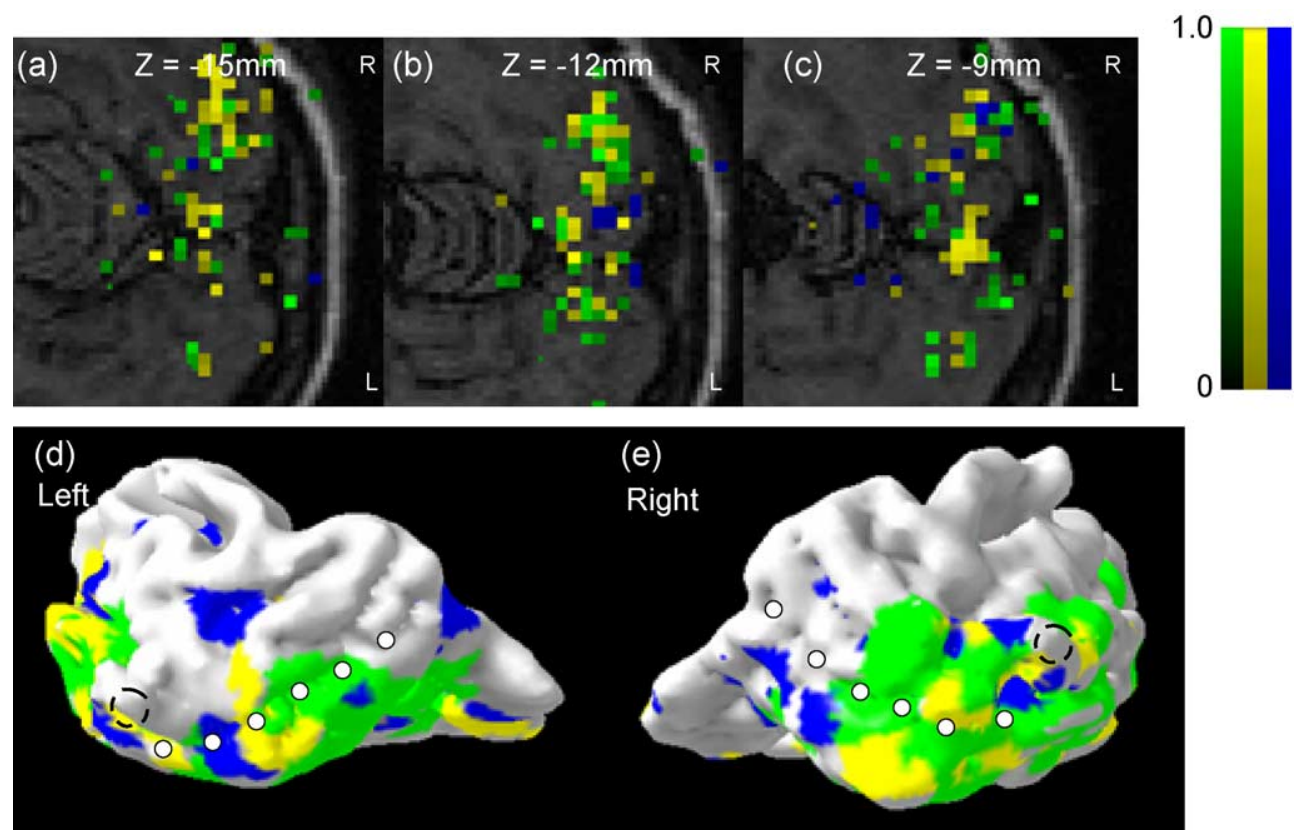

Fig. 5. An activation map for the 1-Hz activation experiment. (a)-(c) show activation on three axial planes with depth $z=-15,-12$ and -9 mm in Talairach coordinates (Talairach and Tournoux, 1988). (d) and (e) show activation on a 3D rendered surface of the left (L) and right (R) occipital cortex to give a clear view of the spatial relation among activated voxels. Colored spots denote voxels showing significant activation change $(|r|>0.35)$ in the experiment (for this observer, $20.6 \%$ of all voxels identified by the spatial localizer). The color codes are the same as in Fig. 2. For this observer, the inter-wedge voxels showing significantly negative correlation with the experimental sequence dropped from $66 \%$ in the $10-\mathrm{Hz}$ adapter experiment to $48 \%$. Hence, the blue spots occupy much less space on this 3 -D rendered image of occipital cortex. 
The modulation of the inter-wedge activation cannot be explained as a result of strong activity in the wedge regions draining blood from the unstimulated regions alone. If this was the case, the inter-wedge activation would have a similar time course as that of the wedge regions. The discrepancies from the bloodsteal predictions therefore suggest that the counter-phase activation is derived from a different mechanism from the in-phase activation. Can this suggestion be tested by a direct manipulation?

\section{Negative BOLD activation and the percept of the twinkle aftereffect}

As a direct test of the blood-steal hypothesis, we changed the adapter flicker frequency from 10 to $1 \mathrm{~Hz}$. If the activity modulation in the unstimulated regions was due to the blood draining from those regions, we should expect the activation pattern with the $1-\mathrm{Hz}$ adapter to be greater than that for the $10-\mathrm{Hz}$ adapter, with a higher proportion of the inter-wedge voxels being in counter-phase with the adapter onset because the stimulation effect in the wedge voxels is higher for $1-\mathrm{Hz}$ square-wave flicker than at $10 \mathrm{~Hz}$ (Campbell and Robson, 1968). On the other hand, it is known that a 1-Hz dynamic surround induces little or no twinkle aftereffect during adaptation in the unstimulated regions (Hardage and Tyler, 1995). Unlike the blood-drainage hypothesis, which predicts that there should be a higher proportion of inter-wedge voxels with counter-phase activation pattern relative to in-phase voxels than at $10 \mathrm{~Hz}$, the aftereffect hypothesis predicts fewer counter-phase voxels at $1 \mathrm{~Hz}$. The experimental results with the $1-\mathrm{Hz}$ adapter clearly showed that, in the inter-wedge regions, the proportion of counter-phase voxels (40-50\%, Fig. 5) is not much different from the proportion of inphase voxels. This proportion represents a significant reduction from the $10-\mathrm{Hz}$ condition where the counter-phase voxels dominated $\left[\chi^{2}(1)=12.84, P<5 \times 10^{-4}\right]$.

\section{The mechanisms underlying the twinkle aftereffect}

Two possible interpretations of the twinkle aftereffect have been proposed. The first interpretation is based on the perceptual filling-in observed in artificially induced scotoma experiments, in which observers often reported that the blank test region vanishes and is replaced by the pattern of the surround after viewing the stimulus for as little as $5 \mathrm{~s}$ (Ramachandran and Gregory, 1991). The idea is that the filling-in process persists upon cessation of stimulation to produce the twinkle aftereffect. The second interpretation suggests that the activation of the visual mechanisms responding to the surround during adaptation produces a suppressive effect on the mechanisms in the unstimulated test regions (Hardage and Tyler, 1995; Ramachandran and Gregory, 1991). This suppression in turn causes a post-stimulus rebound in the test regions. This latter hypothesis is the only one compatible with the extent of the inter-wedge voxels, since no filling-in is reported for such large blank regions.

The counter-phase BOLD pattern is also inconsistent with the filling-in hypothesis because filling-in occurs in less than $5 \mathrm{~s}$ after the adapter onset (Ramachandran and Gregory, 1991; Ramachandran et al., 1993). Given the much longer duration of the adaptation period, we should expect the inter-wedge activation, after allowing a small delay, to be almost in-phase with the adapter. This relation is inconsistent with the activation of the majority of the interwedge voxels, which is essentially counter-phase to the stimulus onset (corresponding to an implausible delay of about $20 \mathrm{~s}$ ). On the other hand, the lateral suppression hypothesis predicts the unstimulated activation to be suppressed throughout the adaptation period and to rebound during the blank test periods. This prediction is fully consistent with our results.

\section{Conclusion}

In conclusion, we have demonstrated a lateral rebound of BOLD activation that correlates with the human percept of activity in unstimulated regions following stimulation of their neighboring regions. This result is not consistent with the idea of blood drainage from unstimulated to stimulated regions during adaptation. Our results indicate that the BOLD signal can be reduced by inhibitory influences on a region of cortex. It also rejects the filling-in hypothesis of the twinkle aftereffect.

\section{Acknowledgments}

This study was partially supported by NIH NEI 13025 to CWT. CLL acknowledges the support of Dr. K.C. Liang of the Psychology Department, National Taiwan University, through funding from the National Science Council, Taiwan. The equipment was partially funded by an Interdisciplinary Equipment Grant of National Taiwan University to Dr. K.C. Liang.

\section{References}

Aubert, A., Costalat, R., 2002. A model of the coupling between brain electrical activity, metabolism, and hemodynamics: application to the interpretation of functional neuroimaging. NeuroImage 17, $1162-1181$.

Buxton, R.B., Wong, E.C., Frank, L.R., 1998. Dynamics of blood flow and oxygenation changes during brain activation: the balloon model. Magn. Reson. Med. 39, 855-864.

Campbell, F.W., Robson, J.G., 1968. Application of Fourier analysis to the visibility of gratings. J. Physiol. 197, 551-566.

Engel, S.A., Glover, G.H., Wandell, B.A., 1997. Retinotopic organization in human visual cortex and the spatial precision of functional MRI. Cereb. Cortex 7, 181-192.

Friston, K.J., Holmes, A.P., Worsley, K.J., Poline, J.P., Frith, C.D., Frackowiak, R.S.J., 1995. Statistical parametric maps in functional imaging: a general linear approach. Hum. Brain Mapp. 2, 189-210.

Hardage, L., Tyler, C.W., 1995. Induced twinkle aftereffect as a probe of dynamic visual processing mechanisms. Vision Res. 35, 757-766.

Hu, X., Le, T.H., Ugurbil, K., 1997. Evaluation of the early response in fMRI in individual subjects using short stimulus duration. Magn. Reson. Med. 37, 877-884.

Kwong, K.K., Belliveau, J.W., Chesler, D.A., Goldberg, I.E., Weiskoff, R.M., Poncelet, B.P., Kennedy, D.N., Hoppel, B.E., Cohen, M.S., Turner, R., Rosen, B., Brady, T.J., 1992. Dynamic magnetic resonance imaging of human brain activity during primary sensory stimulation. Proc. Natl. Acad. Sci. U. S. A. 89, 5675-5679.

Logothetis, N.K., Pauls, J., Augath, M., Trinath, T., Oeltermann, A., 2001. Neurophysiological investigation of the basis of the fMRI signal. Nature 412, 150-157.

Menon, R.S., Ogawa, S., Strupp, J.P., Anderson, P., Ugubil, K., 1995. BOLD based functional MRI at 4 Tesla includes a capillary bed contribution: echo planar imaging correlates with previous optical imaging using intrinsic signals. Magn. Reson. Med. 33, 453-459.

Ramachandran, V.S., Gregory, R.L., 1991. Perceptual filling in of artificially induced scotomas in human vision. Nature 350, 699-702.

Ramachandran, V.S., Gregory, R.L., Aiken, W., 1993. Perceptual fading of visual texture borders. Vision Res. 33, 717-727. 
Röther, J., Knab, R., Hamzei, F., Fiehler, J., Reichenbach, J.R., Büchel, C., Weiller, C., 2002. Negative dip in BOLD fMRI is caused by blood flow-oxygen consumption uncoupling in humans. NeuroImage $15,98-102$.

Shmuel, A., Yacoub, E., Pfeuffer, J., Van de Moortele, P.F., Adriany, G., Hu, X., Ugurbil, K., 2002. Sustained negative BOLD, blood flow and oxygen consumption response and its coupling to the positive response in the human brain. Neuron 36, 1195-1210.

Stehling, M.K., Turner, R., Mansfield, P., 1991. Echo-planar imaging: magnetic resonance imaging in a fraction of a second. Science 254, $43-50$.

Talairach, J., Tournoux, P., 1988. Co-Planar Stereotaxic Atlas of the Human Brain: 3-Dimensional Proportion. Thieme Medical, Stuttgart.

Tootell, R.B., Hadjikhani, N.K., Vanduffel, W., Liu, A.K., Mendola, J.D., Sereno, M.I., Dale, A.M., 1998. Functional analysis of primary visual cortex (V1) in humans. Proc. Natl. Acad. Sci. U. S. A. 95, 811-817.

Wandell, B.A., Chial, S., Backus, B.T., 2000. Visualization and measurement of the cortical surface. J. Cogn. Neurosci. 12, 739-752. 